\title{
CAPITALISMO, ECOCIDIO, GENOCIDIO: EL CLAMOR DE LOS PUEBLOS INDIGENAS*
}

\section{Giulio Girardi}

$\mathrm{M}$ e he permitido reformular el título de mi ponencia, para que se corresponda más a su contenido, de la forma si guiente: "capitalismo, ecocidio, genocidio: el clamor de los pueblos indígenas". Título que a un científico social le parecerá quizás demasiado emotivo y dramático. Yo creo que sí, es emotivo y dramático, pero no demasiado. Porque los que pretendemos abordar, son problemas de vida y muerte: para la naturaleza, para muchos pueblos del mundo, para toda la humanidad. Por primera vez la especie humana se encuentra brutalmente ante el problema de su sobrevivencia y con la posibilidad concreta de decidir su destino.

Esto significa que la ecología no es un tema particular entre muchos otros, sino una problemática que involucra la organización económica del mundo y en último término toda la civilización occidental, los valores y la cultura que la inspiran, el modelo de democracia que ella ostenta.

La ecología, terreno de una toma de partido en los grandes conflictos sociales y mundiales

Fl carácter dramático del problema aparece más evidente, cuan do se percibe que la ecología no es una disciplina neutral (como,

* Ponencia presentada en el II ${ }^{\rho}$ Foro religioso popular sobre "Cristianismo, Justicia, Ecología", Vitoria-Gastauz (España) 6-8 de mayo de 1994. 
por lo demás no lo es ninguna de las ciencias sociales), sino que implica una toma de partido en los grandes conflictos ideológicos de nuestra época, y en primer lugar en el conflicto Norte-Sur.

Los movimientos ecologistas o verdes de Europa no se ubican fácilmente en el espectro político (izquierda, derecha, centro) justamente porque suelen estar divididos entre sí por las opciones políticas que inspiran consciente o inconscientemente sus análisis y sus alternativas. Hay una búsqueda ecológica que es parte de la cultura dominante liberaldemócrata. Ella denuncia las devastaciones del ambiente, provocadas por la orientación de la economía, pero sin ponerlas en relación con la misma lógica del sistema capitalista y por tanto sin llegar a cuestionarla radicalmente. Por tanto, las alternativas que propone sólo pretenden ser formas de perfeccionamiento del sistema, contribuyendo a su racionalización y estabilidad.

Entre los ejemplos más significativos de este tipo de ecología, quiero citar el Informe Bruntland, publicado en 1987 por la comisión mundial para el ambiente y el desarrollo, nombrada por las Naciones Unidas; y la Cumbre de la tierra, celebrada en Río de Janeiro, en junio de 1982.

El momento más "avanzado" en la integración de la ecología a la cultura dominante y por lo tanto a la economía de mercado se da cuando la ecología se convierte en una empresa lucrativa. Por ejemplo, cuando se plantea la exigencia de que los coches, para dejar de contaminar el ambiente, sean provistos de aparatos catalizadores y por tanto la producción de estos aparatos y de carros aptos a recibirlos se convierte en un negocio. Asimismo en ciertos sectores, el producto "más ecológico" llega a ser más competitivo y por lo tanto más rentable.

Pero lo que ubica más decididamente la ecología en la cultura dominante es el silencio que guarda sobre la responsabilidad de las transnacionales, de los organismos financieros internacionales, como el Banco Mundial o el Fondo Monetario Internacional y de las grandes potencias del Norte en la contaminación y destrucción del ambiente en los países del sur; es también la tendencia a concentrarse, en sus análisis y su búsqueda de alternativas, sobre aspectos particulares y locales, sin llegar a un enfoque global, lo que le permite evitar el problema de la contradicción entre la lógica 
productivistica y competitiva del gran capital y la defensa del ambiente. Queda así relegada la interrogante central de la ecología, que concierne justamente la posibilidad de salvaguardar la naturaleza dentro del presente orden económico mundial.

Nuestro lugar antropológico y ecológico: la praxis liberadora de los pueblos indigenas

osotros, en cambio, pretendemos aquí contribuir a la elabo
ración de una ecología que se inserte en una cultura alternativa y liberadora. Es decir que reconocemos desde el primer momento el carácter no neutral sino partidario de nuestro enfoque. $Y$ nos ubicamos para este fin en el lugar antropológico que es la praxis liberadora de los oprimidos, que se convierte así también en "lugar ecológico": los análisis y las alternativas que vamos a proponer pretenden justamente reflejar el punto de vista de los oprimidos como sujetos.

Pero, ¿de qué oprimidos en concreto? Entre los múltiples grupos y pueblos, hemos escogido a los pueblos indígenas concientizados, que manifiestan una sensibilidad excepcional a la cuestión ecológica. Por varias razones. En primer lugar, porque la vida y la sobrevivencia física y cultural de estos pueblos están profundamente vinculadas a la naturaleza. Ellos experimentan en carne propia el efecto devastante que produce el "progreso de la civilización" sobre su ambiente de vida y por eso mismo sobre su vida. Experimentan la coincidencia e interacción entre destrucción del ambiente y destrucción del pueblo, entre ecocidio y genocidio. Ecocidio y genocidio que contribuyen esencialmente a definir el sentido del conflicto fundamental Norte-sur.

En esta experiencia de muerte, ellos perciben además la amenaza que pesa sobre el futuro de toda la humanidad, si no se verifica a corto plazo una inversión de tendencia histórica. De aquí la convicción de que ellos, al defender la naturaleza, no están sólo luchando por su sobrevivencia, sino que están asumiendo la responsabilidad del destino de la humanidad.

Es para evidenciar el carácter dramático de la problemática ecológica planteada por los indígenas en términos de vida y muerte que hablamos aquí de su clamor, de su S.O.S. ante el ecocidio y 
el genocidio, del cual son las principales víctimas; de su llamada de atención a toda la humanidad, obnubilada por su "progreso", sobre el abismo hacia el cual está caminando.

Otra razón que nos empuja a valorar particularmente en esta reflexión el punto de vista de los indígenas concientizados, es que ellos poseen un patrimonio cultural y religioso de relaciones armoniosas y cariñosas con la naturaleza (la Madre Tierra, el Padre Sol, la Abuela Luna), que representan un enorme recurso para la humanidad, hasta ahora totalmente ignorado, en su búsqueda de alternativas a la civilización actual. Cuando los pueblos indígenas afirman con orgullo que su cosmovisión tiene ideas y valores, capaces de brindar un aporte a la solución de la crisis de civilización, ellos insisten particularmente en su manera de concebir la relación con la naturaleza. Por supuesto, estarán dispuestos a valorar este aporte sólo los que creen sinceramente en la sabiduría de los oprimidos que emergen a la dignidad de sujetos históricos; y que, por el otro lado, no consideran todas las culturas precolombinas o precapitalistas como inferiores con respecto a la cultura capitalista y por lo tanto definitivamente descalificadas por el "progreso".

Una tercera razón, que nos empuja a valorar en nuestra reflexión el aporte de los pueblos indígenas, es que una ecología militante, como pretende ser la nuestra, no puede contentarse con indicar los caminos de la alternativa, sino que debe preocuparse por identificar los sujetos capaces de realizarla. Ahora el movimiento indígena, continental y mundial, es indudablemente uno de los grandes sujetos comprometidos en la lucha ecológica. Un sujeto, por lo demás, profundamente consciente de que su lucha se tiene que llevar unitariamente con todos los sectores populares del continente y del mundo.

La perspectiva de los pueblos indígenas, la sacaremos de un conjunto de documentos de alcance continental y mundial, producidos por la campaña continental 500 años de resistencia indígena negra y popular (1989-1992), por el primer encuentro continental de los pueblos indios (1991) y por las dos cumbres mundiales de los pueblos indígenas, que Rigoberta Menchú ha convocado en el año 1993. ${ }^{1}$

En estos documentos, líderes indígenas de Abya Yala y de todo 
el mundo, brindan análisis rigurosos de la destrucción ecológica y proponen alternativas para invertir la tendencia histórica. Análisis y alternativas que abordan la ecología no como un sector aislado de la sociedad y el mundo, sino como estrechamente vinculada con los otros aspectos de la sociedad, particularmente el político y jurídico, el económico, el cultural y religioso. En el centro de los análisis y de las alternativas se encuentran justamente la denuncia de la contradicción radical entre el gran capital y la defensa del ambiente; y la convergencia entre destrucción del ambiente y exterminio de los pueblos indígenas, en otras palabras entre ecocidio y genocidio.

Queremos ahora desde el punto de vista de los indígenas, $1^{\text {9) }}$ abordar el análisis de la relación entre capitalismo y defensa del ambiente, evidenciando la contradicción entre ellos; 29) explorar los caminos de una alternativa ecológica al capitalismo.

\section{La contradicción capitalismo - naturaleza}

$\mathrm{F}^{1}$ análisis hace hincapié en la experiencia de cada pueblo indí Egena al interior de su "estado nacional", que lo lleva a denunciar una forma de "colonialismo interno", es decir un sistema nacional de dominación, marcado por un racismo político y jurídico, cultural y religioso, económico y ecológico. A su vez el colonialismo interno remite al "colonialismo externo", es decir al sistema mundial de dominación y la relación Norte-Sur.

Al calificar la civilización occidental en su conjunto como racista, el movimiento indígena negro y popular está dando, sobre la base de su enorme experiencia histórica, un aporte significativo a la interpretación del racismo y a la concientización de la humanidad sobre este tema crucial. Porque la noción corriente de racismo se refiere a las manifestaciones "monstruosas" y "criminales" del complejo de superioridad de una raza sobre las otras: como es el caso del nazismo o, en la Europa de hoy, de los naziskin alemanes o de los lepenistas franceses. Sin embargo, el análisis de la Campaña 500 años lleva a descubrir una forma de racismo más universal y más oculta, porque forma parte de la ideología que ha justificado las conquistas y colonizaciones realizadas por los países del Norte, y por tanto de las estructuras de dominación que ellas han engendrado. Un racismo que ha llegado a formar parte de lo normal. Y que como tal está interesado por las grandes mayorías de los ciu- 
dadanos del Norte y quizás también del Sur.

\section{Raíces del colonialismo y el racismo: el capitalismo transnacional}

Profundizando luego el análisis, los delegados identifican las beral percibido como sistema de dominación económica transnacional, y por tanto como negación de la autodeterminación de los sectores indígenas, negros y populares.

"Los distintos modelos de colonización han mantenido una constante en cuanto a los atropellos, agudizados por la implantación de sistemas racistas, que se fortalecen con el modelo económico neoliberal". (Managua, p. 23) "El neoliberalismo constituye una mera expresión del neocolonialismo y está dirigido a afianzar las estructuras de dependencia de los pueblos de nuestra América". (Quetzaltenango, p. 40).

Es significativo que, en el II Encuentro Continental de la Campaña sea la comisión Derechos Humanos "derechos indios" la que denuncia más vigorosamente los efectos devastadores del sistema: "La imposición de la llamada "democracia" del sistema capitalista neoliberal, saqueador de recursos, genocida, etnocida, que por medio de la deuda externa chupa la sangre de nuestros pueblos y genera hambre, miseria, analfabetismo, desnutrición y muerte". (p. 43). Poniendo está denuncia en primera plana, la comisión identifica en la misma lógica despiadada del capitalismo una violación masiva de los derechos humanos y en particular de los derechos indios; y la raíz de innumerables otras violaciones. De tal manera denuncia la esencia racista del sistema capitalista neoliberal.

Entre las consecuencias más graves de la lógica capitalista, los indígenas señalan la desnutrición y el hambre: "La tenaz introducción de monocultivos, el destrozo de nuestra rica y variada producción y alimentación tradicionales, la depredación de nuestros manglares y selvas, han provocado la introducción de cambios en sistemas alimenticios que generan la creciente desnutrición de nuestros pueblos". (Quito, p. 262) "Se imponen modelos de desarrollo económico ajenos a nuestra cultura y necesidades en base a monocultivos, que nos obligan a producir lo que les interesa a monopolios internacionales" (p. 262-3). 
No sorprende, después de estos análisis, el pronunciamiento de los jóvenes, quienes interpretan el sentimiento general: "Rechazamos la imposición del modelo económico neoliberal, como una forma de opresión y colonización de nuestros pueblos". (Quetzaltenango, p. 61).

Si la opresión de cada sector popular remite al colonialismo interno, este a su vez remite al colonialismo externo del "imperio capitalista". La "dominación interna, asegura el II encuentro continental, es consecuencia del colonialismo histórico y del neocolonialismo, lo cual significa la agresión económica, política, social, cultural, moral y espiritualmente destinada a perpetuar desde adentro el orden injusto sobre nuestros pueblos". (p.36) "En nuestro continente, los gobiernos que se autodenominan democráticos... han sido fieles títeres de las potencias mundiales que se reparten el mundo, trayendo como consecuencia la pérdida de la soberanía y de los valores religiosos, de la cultura, la violación de los derechos humanos y la corrupción institucional". (p. 33). Entonces el continente latinoamericano se presenta como un conjunto de "estados dependientes del imperio capitalista". (p. 48).

Es particularmente insistente la denuncia del imperialismo norteamericano: "La lucha por la tierra y la liberación nacional y social han sido duramente reprimidas por el imperialismo yanqui y las oligarquías criollas. Millones de campesinos y nacionalidades indígenas han caído víctimas de la violencia y el terrorismo de estado insertados en la doctrina de seguridad nacional y ordenados por el Pentágono". (Bogotá, p. 294).

Sin embargo, las grandes potencias suelen intervenir cada vez más bajo el mantel de los organismos financieros internacionales y de las transnacionales. Es el entrelazamiento entre las grandes potencias, las transnacionales y los organismos financieros internacionales que constituye, según este análisis, el bloque dominante del Norte, y que le da un contenido no ideológico, sino trágicamente realista a la palabra "imperialismo". Al imperialismo así entendido se le considera como la causa fundamental de la opresión de los pueblos del Sur y particularmente de los indígenas y negros y de los sectores populares. El imperialismo así entendido es, entonces, al mismo tiempo una estructura racista y el fundamento del racismo mundial. 
"Cargamos sobre nuestras espaldas los fardos de una deuda creciente, que no es más que nuestra riqueza convertida en préstamo. Como antes, de nuestras vetas sale el oro; de nuestras entrañas, el petróleo; de nuestro sudor, los capitales; de nuestros sueños las pesadillas de la represión y el hambre.

“Los nuevos pregoneros, en su arrogancia, pretenden ocultar una realidad evidente ante nuestros ojos: el capitalismo no tiene soluciones para garantizar un mundo de paz y de justicia social. (Managua, pp. 40-41).

En una palabra: el sistema capitalista, culpable del genocidio perpetrado por las conquistas y colonizaciones del pasado, como del genocidio permanente que se produce en la actualidad es el problema fundamental de la humanidad: no puede entonces de ningún modo brindarle soluciones. Así el movimiento indígena negro y popular percibe el "triunfo del capitalismo" proclamado, después del "derrumbe del comunismo", por la ideología liberal-demócrata.

Dominación capitalista y destrucción del ambiente: el racismo ecológico

$\mathrm{F}^{\mathrm{n}}$ el análisis del sistema capitalista y de las relaciones de do mensión ecológica: es decir la denuncia de la contaminación y destrucción del ambiente, como consecuencia de la ocupación de territorios importantes especialmente por parte de las transnacionales. Donde, coherentemente con la lógica del sistema, el afán de lucro prevalece sobre las exigencias de la vida y el derecho de los pueblos. Este análisis es significativo también como enfoque del problema ecológico desde el punto de vista de los pueblos del Sur. El permite descubrir, como lo planteamos desde el primer momento, que la ecología no es neutral y que existe en la organización del mundo lo que el III encuentro continental empieza a llamar "racismo ecológico". (Managua, p. 14). Los líderes indígenas pretenden además introducir en el lenguaje jurídico el término de ecocidio. "Al igual que lo hiciera la Conferencia sobre "Medio Ambiente y Poblaciones Indígenas", que tuvo lugar en Santiago de Chile en 1992, insistimos en la necesidad de tipificar, a nivel nacional e internacional, el "Delito de Ecocidio", por la relación directa que este tiene con el etnocidio y el genocidio. (Rigoberta Menchú, Reflexiones ante la II 
Cumbre).

Este enfoque de la problemática ecológica presenta algunas características, que intentamos proponer sistemáticamente.

1. El análisis de cada situación tiene como referencia la lucha de los pueblos involucrados, con particular atención a los indígenas, pero sin olvidar a los negros y a los otros sectores populares.

2. El análisis evidencia la convergencia e interacción entre destrucción del ambiente y destrucción de los pueblos, particularmente indígenas, entre ecocidio y genocidio. "El equilibrio y la dinámica de vida en nuestros territorios son destruidos por la explotación voraz de los recursos y la utilización de diferentes elementos químicos que no sólo envenenan el subsuelo sino también al hombre y todo ser viviente". (Quito, p. 262).

"Durante los últimos años nuestra tierra ha sido robada por diferentes gobiernos y por las empresas transnacionales, explotadas de los recursos que se hallan en ella. Estos recursos, por cierto, son necesarios para nuestra sobrevivencia. El resultado de esta explotación no es el desarrollo, sino la muerte de nuestras comunidades, a causa de la contaminación tóxica de la tierra y el agua, y de las políticas etnocidas y genocidas por parte de los gobiernos manipuladores que tratan de destruirnos". (p. 275).

Me parece que esta convergencia entre ecocidio y genocidio autoriza a proponer la palabra de ecogenocidio.

3. La destrucción del ambiente provoca la destrucción física y cultural de los pueblos en primer lugar, porque viola su derecho de autodeterminación política, económica, ecológica y cultural; además, destruye sus fuentes de alimentación, provoca desplazamientos violentos etc. Fundado en este análisis el movimiento demanda "el fin de políticas de desarrollo que incrementan la destrucción de la madre naturaleza y nuestra cultura" (Managua, p. 22) y exige que "el desarrollo de los pueblos indígenas sea promovido por los mismos pueblos en base a su propia cosmovisión. Lo cual evitará la desforestación, la destrucción ecológica y la contaminación de nuestra naturaleza, nuestros ríos, mares, flora, fauna suelo y subsuelo, como es el caso específico de los Pueblos Indígenas de Alaska". (Quetzaltenango, p. 47). Asimismo los Delegados resuelven "con- 
denar a los buscadores de minerales que desplazan a los pueblos indígenas de Amazonas, que contamina su medio ambiente como en el caso de los yanomamis y otros territorios indígenas, así como las grandes transnacionales, que desforestan el área del amazonas". "Decimos no al desplazamiento de las once naciones indígenas ubicadas al este de Canadá, donde se pretende construir 27 represas hidroeléctricas, afectando un área igual al territorio de Francia". (p. 62).

4. La destrucción de la naturaleza, que amenaza, a corto y mediano plazo, la sobrevivencia de los pueblos indígenas, amenaza a largo plazo la sobrevivencia de la humanidad.

5. Raíz del ecocidio y del genocidio es la lógica del gran capital o del mercado total, que reduce la tierra a mercancía. "La tierra no debe ser tratada y manejada como una mercancía: no se puede vender o no puede un hombre apropiarse del medio fundamental que garantiza la sobrevivencia no sólo de una población, sino de la humanidad. El atentar contra el equilibrio, romper su armonía significa atentar contra el desarrollo armónico y natural de la humanidad". (Quetzaltenango, p. 48).

6. Culpables del ecocidio y genocidio son los latifundistas que concentran en sus manos extensiones crecientes del territorio; las transnacionales, que explotan y saquean los recursos minerarios y forestales, implementan la agricultura con química venenosa, contaminan la tierra, el agua, la atmósfera y las mismas personas. Desde su primer Encuentro Continental, la Campaña denuncia el aspecto ecológico de la agresión: "Hoy, después de 500 años, la tierra continúa concentrada en manos de la gran burguesía agraria, comercial y financiera, terratenientes y grandes multinacionales fundamentalmente yanquis. Estas últimas continúan saqueando nuestros recursos naturales, renovables y no renovables, oro, plata, petróleo, etc. con las consecuencias económicas, sociales, políticas y ecológicas que atentan contra la continuación de la vida en el mundo". (Bogotá, p. 293).

Los Delegados deciden por lo tanto "rechazar a las transnacionales que sirven a los interequetzaltenangoses de los grandes capitalistas financieros... dejando en los campos sólo desolación y muerte". (p. 297). Asimismo rechazar "la agricultura implementada por las transnacionales con química venenosa tan dañina para la esta- 
bilidad ecológica, económica y la autonomía de las comunidades" (p. 295).

7. Existe una constante complicidad entre los grandes poderes económicos y los gobiernos: que venden espacios naturales y reservas ecológicas a transnacionales, confiscan en el nombre del "bien público" territorios indígenas para luego entregárselas a las transnacionales; autorizan y defienden la instalación de estas empresas; construyen ellos mismos centrales hidroeléctricas; contribuyen al desplazamiento forzoso de comunidades indígenas; reprimen violentamente las organizaciones indígenas y populares; con su política contrainsurgente y guerrista destruyen los bosques, la fauna y la flora; en todos estos campos desconocen y pisotean el derecho de los pueblos indígenas a la autodeterminación. Se denuncia, por ejemplo "la confiscación por el Estado, en nombre del "bien público", de territorios indígenas, que se declaran parques nacionales y luego se entregan a as transnacionales. Se citan dos ejemplos: referidos uno a la política de Estados Unidos en Nuevo México, con.los pueblos Zuni, Mimbres, Abó, Atrisco y Quari; otro a la política de Ecuador, en los territorios amazónicos. (Quetzaltenango, p. 43) "La política contrainsurgente de los gobiernos y consecuencias de las guerras nos han destruído los bosques y la tala nos agrava la desaparición de la fauna y la flora, convirtiéndolos en desiertos, causando sequía y que nuestros ríos se sequen. (Managua, p. 30).

8. Las responsabilidades fundamentales pesan sobre las grandes potencias del Norte, especialmente sobre Estados Unidos, y sobre los organismos financieros internacionales, que imponen su política destructora a los Estados del Sur; que además los chantajean con los mecanismos de la deuda externa, considerando la entrega del ambiente como forma de pago. Se denuncia particularmente "el cambio de deuda externa por "ecología", nueva práctica etnocida que se da en todos los países... Como ejemplo de estos casos extremos tenemos la lucha de los indígenas por el territorio ACHE en Paraguay, por haberse declarado reserva del Banco Mundial; como también el caso de Honduras en el que la MOSQUITIA (declarado pulmón de la humanidad) está amenazada por la voracidad de las transriacionales, como es la Petrolera Shell..." (Quetzaltenango, p. 43). 
9. El movimiento denuncia el carácter discriminatorio y racista de las políticas ecológicas con respecto a las mayorías pobres y a los pueblos del Sur. "Las grandes mayorías (pobres) cargamos a las consecuencias del deterioro ambiental". Se propone por tanto "presentar una moción de protesta frente a los proyectos de depósitos de basura tóxica en los pueblos de América Latina"; pero sobre todo "protestar y presionar a los gobiernos sobre problemáticas y tenemos como pueblo, responsabilizando a los gobiernos imperialistas y aquellos que fomentan prácticas discriminatorias a los pobres, denunciando los verdaderos culpables del deterioro del medio ambiente". (Managua, p. 30).

10. Como lógica consecuencia de esta denuncia, el movimiento rechaza la deuda externa y reivindica la deuda ecológica que las potencias del Norte tienen con los pueblos del Sur. Tenemos que "negarnos a pagar la deuda externa, porque no debemos nada, sino que hemos despojados de nuestras riquezas". (p. 30). En el mismo encuentro, los delegados de la Región Andina introducen una nueva categoría de análisis, al denunciar la deuda ecológica de las potencias del Norte: "Desconocemos la deuda externa impuesta a los pueblos, reivindicando la deuda histórica y la deuda ecológica que las metrópolis tienen con nosotros". (p. 7).

11. En último término, bajo la acusación de ecogenocidio cae todo el orden mundial, fundado en el neoliberalismo económico, político y cultural; cae, en otras palabras, la civilización occidental. La acusación no se refiere sólo al pasado, sino sobre todo al presente y también al futuro. Porque la lógica del gran capital, que provoca necesariamente estos crímenes, continúa cínicamente su camino de muerte. Los delegados aseguran que "este ataque masivo neoliberal no es más que la continuación del condenable mal trato de recursos materiales y humanos como son el indebido usufructo de nuestros recursos naturales y humanos. Nos referimos a la experimentación química y biológica que se ha venido haciendo en países como Honduras y Puerto Rico, donde ahora mismo se desarrolla un experimento con la siembra de coca y armas químicas". (Quetzaltenango, p. 41). Con esta incesante denuncia, el movimiento indígena se convierte en conciencia crítica y profética de nuestra civilización.

Sin embargo, la plena coherencia de la criminalidad con el con- 
junto del sistema hace que ella quede oculta a los ojos de las grandes mayorías. La encubren pues están las propias estructuras económicas y políticas que presentan la dominación como una relación natural y normal; y la cultura colonialista neoliberal, que justifica esta relación. Por eso el movimiento plantea entre las tareas principales de su estrategia la de concientizar al pueblo sobre la función de las transnacionales y del sistema capitalista mundial.

\section{Presupuestos ideológicos del ecogenocidio: el neoliberalismo}

$\mathrm{D}$ escribiendo la civilización occidental, los líderes indígenas, negros y populares denuncian también su ideología. Para entender el sentido de su interpretación de la "ideología occidental", que puede resultar chocante para nuestra sensibilidad europea y cristiana, hay que recordar que ella no se funda en textos oficiales, profanos o religiosos, sino en la práctica histórica de occidente, así como sus víctimas la fueron experimentando a lo largo de estos 500 años y la siguen sufriendo en la actualidad. A la luz de esta experiencia, la ideología occidental les aparece marcada, entre otras cosas, por el individualismo, el economicismo, el racismo y el militarismo.

El individualismo es una concepción de la vida y la sociedad, donde cada persona, grupo social o pueblo persigue sus propios intereses, compitiendo con todos los demás; donde por tanto la ley fundamental es la lucha por la vida con la sobrevivencia del más fuerte.

El economicismo liberal consiste en reconocer como criterio fundamental de las grandes decisiones económicas y políticas las leyes del mercado y por lo tanto la ley del más fuerte, que prevalecen así sobre los derechos humanos, los derecho de los pueblos, los derechos de la naturaleza. La tierra en particular se considera una mercancía y se dispone de ella con este criterio.

El economicismo liberal implica una noción de productividad, definida como capacidad de producir mercancías; por tanto, considera improductivo lo que sirve únicamente a alimentar y proteger la vida. Implica además una concepción del progreso, el desarrollo y la civilización, definidos esencialmente en función de los avances tecnológicos y militares: lo que autoriza a destruir las culturas y civilizaciones "precapitalistas" o primitivas", para permitir a los 
pueblos el acceso a la modernidad.

El economicismo conlleva una interpretación de la relación Norte-Sur cuyo eje es la noción de "deuda externa", (decididamente rechazada, como lo hemos recordado, por los indígenas, que hablan, al contrario de la "deuda histórica" y la "deuda ecológica" del Norte). Se justifica así la entrega de la soberanía nacional de los estados del Sur a los organismos financieros internacionales, las empresas transnacionales y las potencias del Norte.

Así el economicismo liberal engendra el racismo, es decir el complejo de superioridad de los pueblos "más avanzados", que se sienten legitimados a imponerles a los otros sus modelos políticos, económicos y culturales.

El racismo se expresa también en una concepción de la soberanía nacional que le atribuye un poder absoluto al estado y desconoce por lo tanto el derecho de autodeterminación de los pueblos, particularmente de los pueblos "culturalmente atrasados". Se traduce asimismo en una concepción de la unidad nacional, que desconoce el pluralismo cultural y étnico, y define la unidad en función de la cultura dominante. Se justifican así las políticas integracionistas y asimilacionistas con respecto a los indígenas y negros; inclusive la destrucción de las culturas "arcaicas" y los pueblos que las representan.

El colonialismo conlleva inevitablemente el militarismo, es decir la reivindicación, por parte de los pueblos que se consideran superiores, del derecho de imponer su acción civilizadora con las armas: percibiendo en la superioridad militar un signo de superioridad ética y cultural. El militarismo se presenta a menudo bajo el nombre de "doctrina de la seguridad nacional", que permite identificar como enemigos internos a todos los rebeldes y justifica su represión violenta.

La contradicción capitalismo-naturaleza, parte de la contradicción capitalismo-vida

$\mathrm{T}_{\text {popular en denunciar la estructura consenticina ingena negro y }}$ y del mundo. Ahora, una percepción común de la contradicción fundamental es un factor muy importante para la unificación y la 
eficacia de las luchas.

Para caracterizar estos enfrentamientos, los documentos no recurren a la fórmula clásica de "lucha de clases": no porque nieguen la existencia e importancia de esta lucha, sino porque la consideran parte de un conflicto más amplio. Por un lado pues entre las estructuras de dominación cobra gran importancia, como lo hemos señalado, la dimensión internacional y transnacional, que ellos designan a menudo con la palabra "imperialismo".

Por el lado de los sectores oprimidos, el movimiento subraya el papel de los pueblos indígenas y negros y de las grandes mayorías populares, constituídas no sólo por obreros y campesinos, sino por desempleados y trabajadores informales, por mujeres, jóvenes etc., que no se prestan a ser unificados bajo la categoría de "clase explotada" sino más bien bajo la de "pueblo oprimido".

Además en la descripción del conflicto, los análisis no se limi$\tan$ a las relaciones de "explotación", sino que denuncian relaciones de "dominación", "opresión", "marginación", "exclusión", que se ejercen en formas muy complejas e interdependientes, a nivel económico, político, cultural, religioso, etc. y que culminan en el crimen de genocidio y etnocidio. En este análisis, la contradicción capitaltrabajo sigue siendo importante pero se la enfoca más bien como un aspecto de la contradicción fundamental capital-vida del pueblo.

Además, los pueblos oprimidos denuncian la amenaza que el sistema de dominación y explotación, autollamado "nuevo orden mundial", representa no sólo para ellos sino también para la naturaleza y para toda la humanidad, presente y futura. En su perspectiva existe entonces una contradicción Gran capital-Vida, donde la palabra "Vida" tiene el sentido más amplio, por cuanto designa la de los pueblos oprimidos, de la humanidad presente y futura, y de la naturaleza.

\section{Hacia una alternativa ecológica a la civilización occidental}

$\mathrm{F}^{1}$ análisis anterior nos ha llevado a la conclusión de que el E ecogenocidio es el producto natural de la civilización occidental en su conjunto. Por tanto, la defensa de la naturaleza y de los pueblos a nivel cósmico se podrá realizar sólo en el marco de una 
alternativa global, política, económica y cultural. Así, el movimiento indígena, que en el análisis ha actuado como conciencia crítica, se nos presenta ahora con un proyecto de alternativa ecológica, inspirado por su cosmovisión.

\section{La cosmovisión indígena, aporte a una alternativa ecológica.}

Para el movimiento indígena rescatar la cultura no significa sólo 1 redescubrirla sino también revalorarla en la actualidad: es decir, convencerse de que, con respecto a ella, la cultura, la civilización, la religión impuestas por los conquistadores no representan un progreso; y qué, en cambio, su propia cultura es capaz de alumbrar, para los pueblos indígenas de hoy y para los otros sectores populares, los caminos de un nuevo futuro, de libertad y dignidad. Sólo a partir de esta convicción, los indígenas de hoy, y particularmente los jóvenes, pueden llegar a asumir con orgullo en su identidad actual la cultura de los antepasados. De lo que se trata entonces, es de rechazar el presupuesto fundamental, con que los colonialistas de ayer y de hoy, han justificado y justifican la imposición de su cultura, religión y civilización: el de su evidente superioridad. De lo que se trata, no es sólo de reivindicar el derecho de cada pueblo a la identidad, sino también de afirmar la superioridad ética y política de sus culturas; que, por supuesto, puede coexistir con una inferioridad científica, tecnológica y militar.

La valoración de culturas que la ideología occidental denomina "primitivas" o arcaicas" o "precapitalistas" supone una concepción del progreso, del desarrollo, de la civilización, que rechaza el economicismo liberal y se inspira, en la cosmovisión comunitarista indígena.

Valorando este patrimonio, el movimiento indígena asume, ante la crisis de civilización, su responsabilidad en la búsqueda de alternativas: "En el ocaso del siglo XX, en el que se dice que la "civilización" está en su máximo apogeo y los avances tecnológicos han rebasado todo cálculo; cuando los dos modelos antagónicos en el mundo no han logrado solucionar los problemas de la humanidad y peor aún entender y solucionar la problemática de los pueblos indios; las llamadas potencias que se jactan de su desarrollo han profundizado la desigualdad, la ambición, la crisis, la des- 
trucción ecológica, y han puesto en serio peligro el equilibrio del planeta, surge vigorosa con su responsabilidad histórica la posición alternativa del pueblo indio, producto de su clara visión cósmica y de su coexistencia armónica con la naturaleza (cursiva mío)". (Quito, p. 261).

Al presentar su proyecto de resistencia y alternativa, el movimiento manifiesta también su confianza en la posibilidad de realizarla, a pesar del obstáculo tremendo representado por el sistema de muerte. Fundamento de esta confianza no es un poder económico y militar, capaz de contraponerse al bloque imperial, sino un poder moral y político representado por el protagonismo de los pueblos y por la "cosmovisión" o sistema de valores que los inspira. No es, en otras palabras, el derecho de la fuerza sino la fuerza del derecho, la solidaridad y la verdad.

\section{Contenido de la cosmovisión indígena}

- Pero cuál es concretamente el contenido de la "cosmovisión", ¿cuáles son los valores, que ella pretende rescatar hoy? Encontramos en los documentos de la Campaá 500 años una respuesta negativa y una positiva, que se alumbran mutuamente. La respuesta negativa es la contraposición a la ideología colonialista occidental: "El ser indio... significa manejar una visión del mundo que choca con la práctica de la visión colonialista". (Quetzaltenango, p. 38).

El contenido positivo de la cosmovisión se encuentra sintetizado en algunos documentos. Por ejemplo, la Declaración de Quito tiene unos párrafos intitulados Nuestra visión cósmica: "Nuestra concepción de territorio se sustenta en la forma de entender el sentido de lo humano y de la naturaleza así como la interrelación de estos. Las formas organizativas, políticas, económicas, de producción, en fin todos estos elementos que conforman nuestras cultural, están enraizados y orientados por lo comunitario; por ello creemos que la tenencia de la tierra debe ser colectiva; por ello la cultivamos en comunidad y entre esta distribuiremos sus frutos; por ello sí creemos en la solidaridad y por ello nuestros hijos son de la comunidad.

Es este valor de lo comunitario desde el cual entendemos el sentido de lo humano y la posibilidad de todos y cada uno de lograr 
lograr una vida armónica. Asimismo, este convivir fraterno lo es también con los seres, Es decir con la naturaleza.

No nos sentimos dueños de ella; es nuestra madre, no es una mercancía, es parte integral de nuestra vida. Es nuestro pasado, presente y futuro. Creemos que este sentido de lo humano y del entorno no es solamente válido para nuestras comunidades o para los pueblos indoamericanos.

Creemos que esta forma de vida es una opción, una alternativa, una luz para los pueblos del mundo oprimidos por un sistema sustentado en el dominio entre hombres, entre los pueblos, en el dominio de la naturaleza; un sistema donde prima lo individual, donde los derechos de los pueblos son declaraciones incoherentes con la práctica y donde definitivamente se niegan sus derechos a existir, a tener culturas propias, a la autonomía y a la autodeterminación". (Quito, p. 259-260).

Otra síntesis de la cosmovisión indígena, la brinda la memoria del II Encuentro continental, bajo el título de "Ceremonias". Para aclarar el sentido de las ceremonias religiosas celebradas a lo largo del encuentro y su ubicación en la Campaña, se presenta una síntesis de la "cosmovisión" que las inspira. "Por siglos los habitantes de los pueblos originarios que conforman Abya Yala, hemos vivido de acuerdo con todo el sentimiento y creencias que están englobadas en la Cosmovisión, sabiendo que somos parte de un todo en el que todos los elementos que nos rodean son importantes, el Padre Sol, la Abuela Luna, la Madre Tierra, y todos los que la habitamos.

El equilibrio en todas las cosas fue lo que hizo avanzar a nuestros antepasados en la construcción de unas sociedades acorde con lo que los rodeaba, para que nadie ni nada quedara en el olvido.

Es dentro de este espíritu que nuestros abuelos han vivido $\mathrm{y}$ resistido dentro de estos 500 años, no olvidando, preservando y transmitiéndonos esta sabiduría que enriquece nuestra vida y nos hace sentir un todo con todos.

Es por este sentir de la Cosmovisión que nosotras viudas, los huérfanos, los presos, los oprimidos, los torturados, los desaparecidos, los que sufren hambre y persecución, estamos resistiendo y 
encontrando consuelo y apoyo". (Quezaltenango, p. 102).

Estos textos nos permiten identificar las principales características de la cosmovisión indígena. Queremos señalar aquí las que pueden orientar la elaboración de una alternativa ecológica a la civilización occidental.

1. La experiencia religiosa central que esta visión inspira es la de la unidad de la diversidad entre todos los seres: Dios, naturaleza, hombres y por tanto la importancia de todos y cada uno de ellos. Esta experiencia presenta, con respecto a la ideología occidental, dos grandes novedades: que se refieren una a las relaciones entre las personas, la otra a la relación hombre-naturaleza. Por un lado la cosmovisión indígena se contrapone como policéntrica a la ideología etnocéntrica, eurocéntrica y racista de los colonialistas. Por el otro, ella manifiesta la exigencia de superar un antropocentrismo rígido, para reconocer la dignidad de todos los seres de la naturaleza, no sólo por el servicio que le brindan al hombre, como ambiente de su vida, sino también por su valor propio.

2. A la ideología occidental, fundada en una concepción individualista de la persona, la cosmovisión indígena le contrapone la centralidad de lo comunitario. La persona no concibe su vida y su destino sino insertados armónicamente e inspirados por el valor fundamental de la solidaridad. Este principio orienta las relaciones igualitarias entre hombres y mujeres; exige el carácter comunitario de la propiedad de la tierra, de su cultivo, de la redistribución de sus frutos; la pertenencia de los hijos a la comunidad. La solidaridad comunitaria se extiende también a las generaciones futuras: en su lucha por la defensa de la naturaleza, los indígenas de hoy se sienten responsables del futuro y de la sobrevivencia de la humanidad. La solidaridad comunitaria se extiende a todos los seres de la naturaleza; y orienta la visión indígena de la relación entre los pueblos.

El solidarismo indígena se contrapone también al economicismo neoliberal, por cuanto asume la promoción solidaria de la comunidad como criterio de las grandes decisiones políticas y económicas y por eso mismo como criterio de progreso, desarrollo y civilización.

3. La convivencia comunitaria se inspira en los valores éticopolíticoș de autodeterminación, solidaridad, respeto a la diversi- 
dad. La autodeterminación no se impone sólo como un derecho de los pueblos sino también como un deber: el de defender su propia identidad. "Nos proponemos levantar alternativas pluralistas y democráticas a la situación de opresión, discriminación y explotación que padecemos, sobre la base de las formas de organización comunitaria, que nos han legado nuestros antepasados" (Quetzaltenango, p. 7) " $Y$ es que un reencuentro con nosotros mismos, con nuestras raíces preñadas de sentido comunitario y solidario de esperanza y de vida, no puede sino constituir una respuesta desde la historia a los desafios del presente". (p. 8) "Continuar el fortalecimiento de nuestras Organizaciones y Comités Nacionales, tomando como base los principios, valores morales y experiencias de autogobierno de las comunidades". (p. 29). Esta democracia debería recoger, no solamente las formas comunales de autogobierno, sino los valores como la solidaridad, reciprocidad, apoyo mutuo, la transparencia del poder entre otros". (p. 35).

4. La relación entre los pueblos tiene que fundarse en los mismos valores: reconocimiento del derecho de autodeterminación, respeto a la diversidad, solidaridad, igualdad. Sólo a estas condiciones se puede hablar de un "encuentro de culturas".

5. Entre los hombres y la naturaleza tiene que haber una relación de amor, respeto y armonía: "La concepción indígena sobre la tierra se ve enriquecida por los aportes de los movimientos ecológicos... La tierra es la casa de la humanidad..." (p. 48) "El desarrollo de los pueblos indígenas, sea promovido por los mismos pueblos en base a su propia cosmovisión, lo cual evitará la desforestación, la destrucción ecológica y la contaminación de nuestra naturaleza, nuestros ríos, mares, flora, fauna, suelo y subsuelo". (p. 47) En la declaración de Xelajú, se habla de trabajos inspirados por "la fraternidad y el respeto heredado de nuestros ancestros"... sobre "el significado y el valor que para nosotros indígenas, negros y sectores populares tiene la tierra, ligado a la vida y a la defensa del entorno ecológica y de nuestros recursos naturales". (p. 20).

Los distintos aspectos de la cosmovisión indígena en su contraposición a la ideología colonialista neoliberal remiten en último término al derecho-deber de autodeterminación solidaria: es decir que el derecho de autodeterminación no es propio a los pueblos "superiores" sino a todos los pueblos del mundo: por tanto exclu- 
ye la dominación de un pueblo sobre cualquier otro y al contrario impone un ejercicio de la autodeterminación orientado a crear relaciones de solidaridad entre todos los seres: entre Dios y el universo; entre los miembros de cada comunidad y pueblo; entre hombres y mujeres; entre todos los pueblos del mundo; entre la humanidad presente y la futura; entre los hombres y la naturaleza.

Entonces, el clamor de los indígenas no es sólo un grito de alerta; es también un mensaje, antiguo y novedoso, dirigido a la humanidad: con un proyecto de vida y civilización y una estrategia para realizarlo. Eje del proyecto y la estrategia es el ejercicio de la autodeterminación solidaria, a nivel político y jurídico, a nivel económico y ecológico, a nivel cultural y religioso.

Eje de la alternativa ecológica a la civilización occidental: el derecho de autodeterminación solidaria

\section{Autodeterminación solidaria, alternativa al colonialismo}

T a contradicción denunciada por el movimiento indígena, ne gro y popular entre lógica del Gran Capital y Vida tiene una consecuencia evidente, aunque no fácilmente traducible en términos operativos: y es que la respuesta a los problemas fundamentales, empezando por el de la sobrevivencia de los pueblos y de la humanidad, no se puede alcanzar sin quebrar aquella lógica y por lo tanto sin instaurar un nuevo modelo económico y político. No sólo a nivel local, sino también estatal, continental y mundial.

La palabra "alternativa" que vuelve a cada rato en los documentos es, por cierto, muy vaga; pero expresa por lo menos la exigencia de romper con la lógica vigente y de buscar formas nuevas de convivencia y de producción. Más exactamente, se busca una alternativa y un sistema colonialista: y por lo tanto una organización de la sociedad y del mundo fundada sobre el pueblo y los pueblos como sujetos, es decir sobre su derecho de autodeterminación solidaria a nivel político, económico, ecológico, cultural, educativo, religioso.

Al calificarse como solidaria y policéntrica, la autodeterminación popular se contrapone a la autodeterminación etnocéntrica, entendida como justificación de un proyecto imperial de grandeza na- 
cional, fundado en la dominación de otros pueblos.

Esto significa que la liberación de cada pueblo indígena está vinculada a la liberación de todos los sectores populares del mismo estado, pero también a la liberación de los estados nacionales y de todo el continente. La lucha por la autonomía de cada pueblo no se puede separar de la lucha por la soberanía nacional del estado y por la autonomía del continente del dominio imperial del Norte. Indígenas, negros y sectores populares aspiran por lo tanto a solucionar sus problemas propios y a conquistar su autonomía no en forma separada, sino en el ámbito de una "patria grande" multiétnica y multicultural. Por eso, el derecho de autodeterminación solidaria no encuentra un límite en el derecho de los otros pueblos y en los de la humanidad, sino más bien una condición de posibilidad de su propia afirmación.

La autodeterminación así entendida es la esencia de la democracia participativa e implica, además de la participación popular al poder y por lo tanto el ejercicio del poder delegado en relación estrecha con el sujeto popular, la división equitativa de los bienes, el respeto de la vida y de los derechos fundamentales, el reconocimiento de las formas tradicionales de organización comunitaria, etc. Democracia por lo tanto que se contrapone frontalmente al formalismo de la "democracia" liberal, denunciada como violación sistemática, en el nombre del derecho de autodeterminación de los fuertes, del derecho de autodeterminación de los débiles.

\section{Autodeterminación solidaria y alternativa ecológica}

$\mathrm{Z}_{\text {una alternativa a una civilización que está devastando la natura- }}^{1}$ leza y exterminando los pueblos. Ahora, buscando las causas del ecogenocidio las ha encontrado en un sistema colonialista, donde no sólo los pueblos indígenas sino las grandes mayorías populares están excluidas del poder económico real y por lo tanto no pueden ejercer ningún control sobre la tierra y los recursos naturales. En otras palabras, existe un vínculo estrecho entre la destrucción del ambiente y la destrucción de la democracia política y económica.

De aquí la dirección en la que el movimiento indígena, negro y popular busca la alternativa ecológica a la civilización occidental: 
la del derecho de autodeterminación solidaria de los propios pueblos indígenas y de todos los sectores populares. La afirmación del vínculo estrecho entre defensa de la democracia y defensa del ambiente es el corazón del mensaje ecológico de los indígenas y el más estimulante para la búsqueda de los pueblos del Norte.

"Los pueblos indios consideramos vital la defensa y conservación de los recursos naturales actualmente agredidos por las transnacionales. Estamos convencidos de que esta defensa será real si los pueblos indios son los que administren y controlen los territorios donde habitan, bajo principios de organización propios y formas de vida comunitaria". (Quito, p. 235).

“Exigimos a los gobiernos nacionales la suspensión definitiva de otorgamientos de permisos de explotación de recursos naturales renovables y no renovables, al interior de nuestros territorios indios". (p. 266) "Impulsaremos programas alternativos de desarrollo social que garanticen la soberanía, autonomía, unidad, integridad ecológica de nuestros pueblos. Desarrollaremos nuestra propia política económica en base a la utilización armónica de nuestros recursos naturales, orientados primero al mejoramiento de nuestros pueblos, que nos permitan alcanzar de autogestión, dando una nueva alternativa al orden económico internacional". (p. 267).

“La concepción indígena sobre la tierra se ve enriquecida por los aportes de los movimientos ecológicos y las aspiraciones y necesidades de los diferentes sectores y estratos de la sociedad. La tierra es casa de la humanidad, con todos los elementos que favorecen el desarrollo de la plenitud de la vida, por lo que la lucha estaría apuntando a que todos los pueblos y naciones originales ejerzan control y autonomía sobre la biósfera y atmósfera". (Quetzaltenango, p. 48). El desarrollo de los Pueblos Indígenas sea promovido por los mismos pueblos, en base a su propia cosmovisión, lo cual evitará la desforestación, la destrucción ecológica y la contaminación de nuestra naturaleza, nuestros ríos, mares, flora, suelo y subsuelo". (p. 47).

Los líderes indígenas resuelven "asumir los proceso de Autonomía y Autodeterminación sobre nuestros territorios y recursos naturales, para:

- Beneficiarnos con el acceso y derecho al uso racional y cien- 
tífico de los recursos naturales a nuestro alcance: mares, ríos, lagos y lagunas, bosques y subsuelo.

- Difundir los beneficios de la biodiversidad, en la educación y conocimiento de la riqueza curativa natural de nuestras plantas y animales.

- Desarrollar los mecanismos de autodefensa y protección de nuestros territorios y recursos". (Managua, p. 43).

El movimiento pretende defender la naturaleza no sólo contra la agresión de las transnacionales, sino también contra los efectos de las guerras: "Por el profundo respeto que tenemos a nuestra Madre Tierra, no somos parte de guerras fratricidas, ajenas a nuestra cultura; declaramos a nuestros territorios zonas de paz en caso de guerras y conflictos internacionales". (Quito, p. 266).

Esta propuesta lleva a profundizar por un lado la naturaleza del derecho de autodeterminación solidaria; por el otro el sentido de la problemática ecológica. Se evidencia pues en este contexto que el derecho de autodeterminación no tiene como objeto sólo la vida política y económica, sino también, esencialmente, los recursos naturales. Por el otro lado se entiende que una alternativa ecológica estará estrechamente vinculada a una alternativa política económica y cultural, fundada en la afirmación de la autodeterminación popular.

Por cierto, sobre el terreno económico, se hace todavía más difícil brindarle contenidos concretos a aquella autonomía, de la que se proclama la necesidad inderogable. Se trata, por supuesto, de una autonomía que no excluye múltiples dependencias (inevitables en el mundo de hoy), pero si excluye que los centros decisionales de la vida económica estén sustraídos al control popular y que por lo tanto la vida económica y la misma naturaleza esté subordinada a intereses externos. En último término la autonomía es la posibilidad de crear una economía independiente en alguna medida de las leyes del mercado capitalista y de su tecnología.

Si bien es cierto que esta exigencia de alternativa económica y ecológica autónoma no se ha concretado todavía en un modelo técnico practicable, ella sin embargo indica con extrema urgencia una dirección de búsqueda para toda la humanidad; porque el 
modelo económico alternativo no condiciona sólo la autodeterminación de los pueblos, sino su misma sobrevivencia.

\section{Autodeterminación solidaria y reapropiación de la tierra.}

T a autonomía económica y ecológica exige en primer lugar la que nunca a la defensa de la vida. Luchar por la tierra es un terreno fundamental de unidad indígena, negra y popular, donde los indigenas ejercen un papel de vanguardia.

"Las tierras y el pueblo indígenas son inseparables. La tierra es vida y no se puede ni comprar ni vender. Es responsabilidad nuestra cuidarla según la tradición, para garantizar nuestro futuro". (Quito, p. 267) "Reclamamos que se conceda la soberanía y autonomía política de los territorios ancestrales ocupados por las comunidades autónomas". (Quetzaltenango, p. 61).

La importancia vital de esta reivindicación es expresada muy elocuentemente por los obispos guatemaltecos, citados, con letra mayúscula, en la memoria del II encuentro continental: "EL CLAMOR POR LA TIERRA ES, SIN DUDA ALGUNA, EL GRITO MAS FUERTE, MAS DRAMATICO Y MAS DESESPERADO QUE SE ESCUCHA EN GUATEMALA, BROTA DE MILLONES DE PECHOS DE GUATEMALTECOS QUE NO SOLO ANSIAN POSEER LA TIERRA, SINO SER POSEIDOS POR ELLA". (Carta Pastoral Colectiva del Episcopado Guatemalteco, Febrero de 1988, p. 66).

Los indígenas y los campesinos se proponen ejercer su derecho a la tierra de dos formas concretas, que son dos terrenos de lucha unitaria, respectivamente legal e ilegal: la reforma agraria y la ocupación de tierras. La síntesis de sus reivindicaciones es un proyecto de reforma agraria auténticamente indígena y popular, que hasta ahora no se ha dado en ningún país de continente "con excepción de Cuba y Nicaragua". (Bogotá, p. 293). "Durante estos 500 años, hemos sido sometidos por los españoles, portugueses, ingleses, franceses, y en la etapa actual por el imperialismo norteamericano, que, entrelazado con las oligarquías criollas, implementa reformas agrarias que remodelan el sistema capitalista, modernizando la de pendencia sin cambiar la situación económica y social, entrando en contradicciones con los verdaderos intereses de los campesinos 
y territorios de las nacionalidades indígenas". (p. 292). "Ante tal situación reafirmamos nuestro compromiso de seguir fortaleciendo la unidad de los pueblos latinoamericanos, para continuar luchando por una reforma agraria auténtica e integral que garantice el desarrollo propio indígena y la incorporación con los campesinos al proceso de desarrollo económico, político y social hasta lograr la liberación definitiva jugando un papel protagónico en este proceso". (p. 294) Se resuelve por tanto "luchar por una reforma agraria integral y democrática, cambiándo las estructuras de concentración de la tierra, expropiando los grandes latifundios y transnacionales que insertándolos al desarrollo de nuestros países, permita cambiar las condiciones de vida, salud, vivienda, educación, electrificación, asistencia técnica, comercialización, etc." (p. 295)

Pero, conociendo por experiencia secular la resistencia de casi todos los gobiernos a estas reivindicaciones, ellos deciden al mismo tiempo "el fortalecimiento de nuestras organizaciones indígenas y campesinas, para defender los territorios, tomando tierras sin esperar políticas agrarias del Gobierno". (p. 294) "Hacemos un llamado urgente a nuestros pueblos indios a retornar posesión de tierras o territorios comunales, a fomentar la unidad mediante estrategias concretas de una red de intercomunicación y solidaridad, que elimine fronteras y límites de cualquier clase". (Quito, p. 266).

Estas luchas legales o ilegales por la reapropiación de la tierra encuentran su plena justificación en el derecho de los pueblos originarios sobre las tierras que les pertenecian, que les fueron arrebatadas por los conquistadores de Europa y Norteamérica y que les siguen arrebatando las potencias del Norte, las empresas nacionales y transnacionales, los latifundistas, los Estados Nacionales, las iglesias, etc. Supone entonces que los culpables reconozcan sus crímenes, por tanto su deuda histórica y ecológica, y que estén dispuestos a pagarla.

Sin embargo, estas reivindicaciones chocan contra el muro del etnocentrismo, que les impide a los europeos y norteamericanos de hoy, como a sus ancestros, reconocer a los indígenas como sujetos de historia; que por lo tanto les impide percibir en los procesos de colonización una violación sistemática y criminal de sus derechos fundamentales. Además, los colonialistas de hoy se niegan a asumir las culpas cometidas por sus antepasados, aún cuando las re- 
conocen; rechazan la concepción de la responsabilidad, acorde a la cual las culpas de los padres recaen sobre los hijos.

La reivindicación del movimiento indígena, negro y popular se funda, en cambio, sobre una percepción de la continuidad rigurosa entre pasado y presente. "El lenguaje de la historia oficial no sólo coloca un velo sobre el genocidio y saqueo practicado por los europeos a las antiguas civilizaciones de este continente, sino que sigue fundamentando hoy día la expropiación de los derechos ancestrales que tienen los indígenas en sus territorios". (Bogotá, p. 287).

Porque la situación actual de los pueblos ex-coloniales está influida decisivamente por la serie ininterrumpida de agresiones, expropiaciones, injusticias, de las que fueron víctimas. La misma organización económica, política, cultural, religiosa, de la sociedad, que ellos con todo fundamental llaman neocolonialista, es la prolongación de las relaciones coloniales instauradas hace 500 años. Por el otro lado, ellos piensan que el bienestar del cual nosotros gozamos en el Norte, el progreso científico y tecnológico que hemos llevado a cabo son fruto de la acumulación primitiva que hemos realizado depredando y explotando sus riquezas.

Es en el nombre de esta continuidad entre pasado y presente, como ellos piensan poder exigir hoy la reparación de crimenes de los que ellos sufren hoy las consecuencias mientras que nosotros gozamos hoy sus beneficios.

Es urgente que este problema deje de ser considerado un juego intelectual de algunos moralistas, para recobrar su puesto en la conciencia de la humanidad, como una de las cuestiones morales y políticas, que condicionan su futuro.

Este reconocimiento efectivo sería el signo esperanzador de que una nueva historia, fundada en la fuerza del derecho, la justicia, la solidaridad, está empezando.

\section{Conclusión}

F s particularmente evidente en el terreno de la ecología que El punto de vista de los pueblos indígenas puede ser un aporte muy rico, en esta crisis de civilización, a la toma de conciencia y a la búsqueda de la humanidad. En la medida en que nos ayudan 
a descubrir problemas e intereses vitales que tenemos en común, como son en primer lugar los problemas, indisociables, de la naturaleza y la democracia, los indígenas están llamando a una alianza de todos los sectores populares de América Latina, de Europa y del mundo entero, para la formación de un bloque histórico popular, continental y mundial, llamado a contraponerse en perspectiva, al bloque imperial del norte.

Propongo entonces que, como conclusión de nuestro encuentro nos comprometamos a respaldar con este espíritu el Decenio internacional de los pueblos indígenas, proclamado por las Naciones Unidas, del 10 de diciembre de 1994 al 10 de diciembre de 2004. De lo que se trata, es de una importantísima iniciativa, tomada por las dos cumbres de los pueblos indígenas convocadas en 1993 por Rigoberta Menchú y endosada por la ONU, que la aprobó en su asamblea de Diciembre de 1993.

A lo largo del Decenio, el movimiento indígena mundial se propone luchar fundamentalmente a dos niveles:

$1^{2}$. Desarrollar una intensa acción diplomática ante las Naciones Unidas y ante cada uno de los Estados donde viven sus pueblos, para conseguir el reconocimiento oficial de sus derechos, especialmente con la aprobación del "proyecto de declaración universal de los derechos de los pueblos indígenas" preparado por el Grupo de trabajo sobre poblaciones indígenas de las Naciones Unidas.

$2^{2}$. Lanzar una campaña de concientización de los propios pueblos indígenas sobre sus derechos; y de concientización de la humanidad sobre la marginación histórica de los pueblos indígenas y sobre los problemas que ellos plantean con respecto a su propio futuro y al futuro de la humanidad.

Mi propuesta es que nos comprometamos a respaldar el Decenio, entendiéndolo no sólo como un periodo de solidaridad más activa y beligerante, sino también como un tiempo de concientización de la humanidad sobre los crímenes y los riesgos de nuestra civilización y sobre los caminos de la alternativa. En este espiritu el Decenio de los pueblos indígenas tendría que ser también el de cenio de la Madre Tierra Pachamama.

Propongo además que lancemos un llamado a todos los orga- 
nismos de solidaridad internacional del Estado Español, para que, en la. perspectiva del Decenio internacional, decidan crear una coordinación estatal de solidaridad con los pueblos indígenas; vincularla orgánicamente a la "Iniciativa Indigena por la paz" que se ha constituido en México alrededor de Rigobierta Menchú, y que justamente en estos mismos días se está reuniendo en Ciudad de México para elaborar el plan de acción del Decenio.

Nuestro llamado tendría que valorar la fuerte movilización que se ha verificado en el Estado Español, con ocasión del V Centenario, al lado de la resistencia indígena y popular, evidenciando el carácter no coyuntural sino histórico de aquella explosión de conciencia.

Nuestro llamado podría además proponerle a la nueva coordinadora dos objetivos inmediatos:

12. La solidaridad con el EZLN y con todos los indígenas y campesinos de Chiapas, organizando sobre este tema una campaña de contrainformación. En este momento Chiapas puede ser el detonante de una movilización internacional al lado de los indígenas de Abya Yala y de todo el mundo.

$2^{2}$. Respaldar activamente la campaña para el otorgamiento del premio Nobel por la paz a Don Samuel Ruiz, obispo de San Cristóbal de Las Casas, como reconocimiento de su entrega valiente a la causa de los indígenas y de la participación de las comunidades cristianas, de Chiapas, de México y de toda América Latina en la solidaridad con los pueblos indígenas.

Propongo por fin que si tomamos estas decisiones se las comuniquemos ya con un fax a Rigoberta Menchú y a la "Iniciativa Indígena por la paz", asumiendo así oficialmente con ellos este compromiso.

Quiero concluir evocando el clima de esperanza, para los pueblos indígenas y para el mundo, en que se realiza esta movilización, y que encuentra su elocuente expresión en la Declaración final de la II Cumbre: "En esta II Reunión Cumbre de los Pueblos indígenas arribamos a las conclusiones que más abajo se enuncian, en las que expresamos nuestras esperanzas para el futuro de nuestros pueblos, conscientes de que recogen también nuestras aportacio- 
nes para la paz en el mundo y la sobrevivencia de la humanidad en el próximo milenio...

“Han pasado los 500 años de obscuridad y hoy sabemos que este es el tiempo del Nuevo Amanecer que ha de alumbrar el futuro de nuestros pueblos".

\section{Referencias bibliográficas}

1. Nos fundaremos concretamente en los documentos siguientes: 1) Memoria del Encuentro latinoamericano de organizaciones campesino-indígenas, Bogotá, 7-12 de octubre de 1989, Lanzamiento de la campaña 500 años de Resistencia indígena y Popular, recogia en Documentos Indios, Tomo II, por José Juncosa (comp.), Ediciones Abya Yala, Quito-Ecuador, 1992, pp. 283-311; las motivaciones y los objetivos del Encuentro están presentados en el documento de convocación Campaña continental por el autodescubrimiento de nuestra América, Bogotá, mayo de 1989, ibid., Pp. 275-282. Citaremos estos documentos con la sigla Bogotá.

2) Memoria del $I I$ Encuentro continental de la Campaña 500 años de resistencia indígena, negra y popular, Quetzaltenango, Guatemala, 7-12 de octubre de 1991, tomo de 112 Pp., editado por la secretaría operativa, en Guatemala, Noviembre de 1991. Lo citaremos con la sigla Quetzaltenango. Esta memoria se encuentra también en Documentos Indios, tomo II, Pp. 155-218.

3) Memoria del III Encuentro continental de la Campaña 500 años de resistencia indígena, negra y popular, Managua, Nicaragua, 7-12 de octubre de 1992, tomo de 69 Pp., publicado por la secretaría operativa continental, Managua, Nicaragua, sin fecha (1993). La citaremos con la sigla Managua.

4). Del I Encuentro continental de los Pueblos Indios (Quito, 17-21 Luglio 1990) se ha publicado la Declaración de Quito y Resolución del Encuentro Continental de Pueblos Indígenas en Documentos Indios, Tomo I, pp. 231-278. Citaremos el documento con la sigla Quito.

5) De la Primera Cumbre de Pueblos Indigenas (B'okob, Chimaltenango, Guatemala, 24-28 de Mayo de 1993) se ha publicado el Informe Final, 52 pp. Citaremos el Documento con la sigla Chimaltenango.

6) De la Segunda Cumbre de Pueblos Indigenas, pudimos consultar las Reflexiones ante la segunda reunión cumbre de los pueblos indigenas, de la Rigoberta. Menchú, 6 Pp.; el Informe preliminar de relatoria, 34 Pp. y el Informe final. Citaremos estos textos con la sigla Oaxtepec. 